\title{
Topological Lower Bound on Quantum Chaos by Entanglement Growth
}

\author{
Zongping Gong, ${ }^{1,2}$ Lorenzo Piroli, ${ }^{1,2}$ and J. Ignacio Cirac ${ }^{1,2}$ \\ ${ }^{1}$ Max-Planck-Institut für Quantenoptik, Hans-Kopfermann-Straße 1, D-85748 Garching, Germany \\ ${ }^{2}$ Munich Center for Quantum Science and Technology, Schellingstraße 4, 80799 München, Germany
}

(Received 21 December 2020; accepted 16 February 2021; published 19 April 2021)

\begin{abstract}
A fundamental result in modern quantum chaos theory is the Maldacena-Shenker-Stanford upper bound on the growth of out-of-time-order correlators, whose infinite-temperature limit is related to the operatorspace entanglement entropy of the evolution operator. Here we show that, for one-dimensional quantum cellular automata (QCA), there exists a lower bound on quantum chaos quantified by such entanglement entropy. This lower bound is equal to twice the index of the QCA, which is a topological invariant that measures the chirality of information flow, and holds for all the Rényi entropies, with its strongest Rényi- $\infty$ version being tight. The rigorous bound rules out the possibility of any sublinear entanglement growth behavior, showing in particular that many-body localization is forbidden for unitary evolutions displaying nonzero index. Since the Rényi entropy is measurable, our findings have direct experimental relevance. Our result is robust against exponential tails which naturally appear in quantum dynamics generated by local Hamiltonians.
\end{abstract}

DOI: $10.1103 /$ PhysRevLett.126.160601

Introduction.-The principles of causality and conservation of quantum information impose strong constraints on the evolution of quantum many-body systems. In the simplest setting, where space and time are discrete and causality is "strict." the latter can be described by quantum cellular automata (QCA) [1-3], cf. Fig. 1(a) for an illustration. Despite seemingly crude approximations for realistic many-body dynamics, they provide useful models to study different aspects of nonequilibrium physics. For instance, local quantum circuits, a subclass of QCA, recently received significant attention in connection to questions related to quantum chaos and information scrambling [4-16].

In the past decade, much progress has been made in characterizing QCA [17-21], with comprehensive and elegant results obtained in one dimension (1D) [22-26]. In particular, it was first found in Ref. [22] that QCA are characterized by a topological index "ind," which measures the amount of quantum information flowing through the system. Besides its fundamental interest, this result turned out to have practical implications, allowing, e.g., for classifying 2D Floquet phases exhibiting bulk many-body localization (MBL) [27-34].

In light of the intuitive information-flow picture, it is natural to ask whether there exist strict relations between

Published by the American Physical Society under the terms of the Creative Commons Attribution 4.0 International license. Further distribution of this work must maintain attribution to the author(s) and the published article's title, journal citation, and DOI. Open access publication funded by the Max Planck Society. the index and other aspects of the unitary dynamics, related, for instance, to information scrambling as probed by outof-time-ordered correlators (OTOCs) [35-37]. The main difficulty to answer this question lies perhaps in the original definition of the index [22], which was given by the rank of certain operator algebras, lacking an immediate physical interpretation.

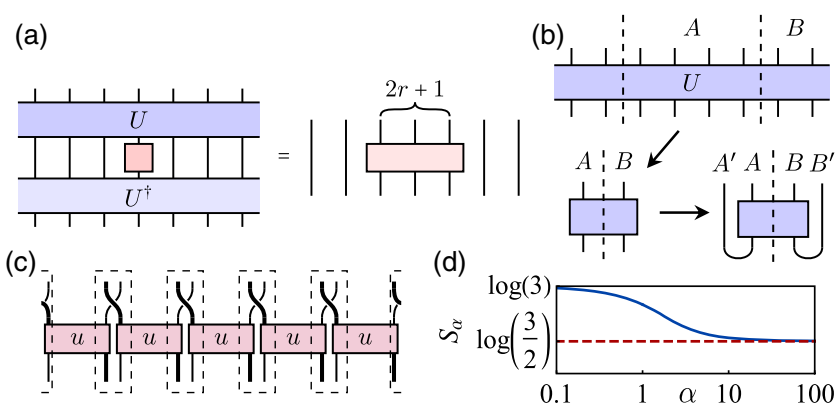

FIG. 1. (a) A range- $r$ 1D QCA $U$ transforms an operator supported on the $j$ th site into one supported on $[j-r, j+r]$. (b) Bipartition $A \sqcup B$ and vectorization of $U$ into the CJS $|U\rangle$ on a doubled Hilbert space [cf. Eq. (4)]. The operator entanglement of $U$ with respect to $A \sqcup B$ is defined as the entanglement entropy of $|U\rangle$ with respect to $A A^{\prime} \sqcup B B^{\prime}$, where $A^{\prime}, B^{\prime}$ are the ancillary qudits associated with $A, B$, respectively. (c) AKLT-like range-1 QCA with local Hilbert space $\mathbb{C}^{p q}$ (dashed rectangles) and ind $=$ $\ln (p / q)$ generated by disjoint unitaries $u$. Here the thin and thick legs correspond to $\mathbb{C}^{p}$ and $\mathbb{C}^{q}$, respectively. (d) Operator entanglement Rényi entropy $S_{\alpha}$ of a single $u$ in (c) with $p=2$ and $q=3$ approaches $\mid$ ind $\mid=\log (3 / 2)$ when $\alpha \rightarrow \infty$, implying the saturation of Eq. (1) for $S_{\infty}$. 
In this work, we prove that there is an equivalent way to express the index as the entanglement of the "vectorized" evolution operator [cf. Fig. 1(b)], which is often called operator-space entanglement entropy [38]. This quantity can be formulated in terms of any Rényi entropy, is computed locally, and closely reflects the intuitive interpretation of the index based on quantum-information flow. Inspired by this definition, we derive our main result,

$$
S_{\alpha} \geq 2 \mid \text { ind } \mid,
$$

where $S_{\alpha}$ is the Rényi entropy of order $\alpha$ of the evolution operator, and where $\alpha \in[0, \infty]$. This result establishes a link between two seemingly unrelated topics, proving that there exists a topological lower bound on the value of wellstudied dynamical quantities. In particular, based on the known relation between $S_{2}$ and the averaged OTOCs [39], we will interpret this inequality as a lower bound on quantum chaos. As an immediate application, Eq. (1) allows us to establish rigorously that any sublinear entropy growth behavior in $1 \mathrm{D}$, including MBL, is not compatible with a nonvanishing index. Thanks to measurability of the Rényi entropy, our results should be experimentally observable.

Index of $1 D Q C A$. - We consider general range- $r$ QCA $U$ defined on a periodic qudit chain with local Hilbert space $\mathbb{C}^{d}$ and size $N$. As shown in Refs. [1,23], by grouping (or "blocking") at least $r$ adjacent sites into one (such that the coarse-grained QCA has size $2 l \leq N / r$ and range 1), we can represent $U$ as [cf. Fig. 2(a)]

$$
U=\left(\bigotimes_{x=1}^{l} v_{2 x-1,2 x}\right)\left(\bigotimes_{x=1}^{l} u_{2 x, 2 x+1}\right),
$$

(a)

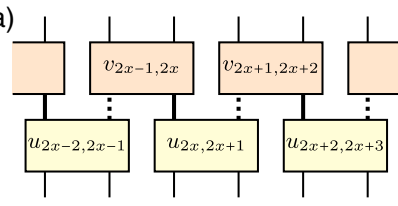

(c)

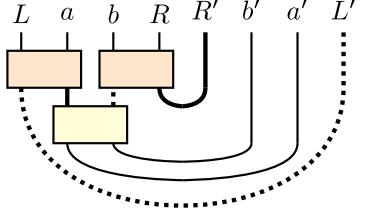

(b)

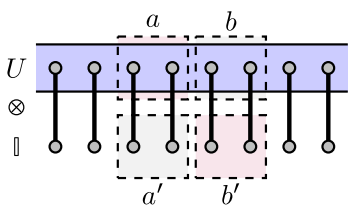

(d)

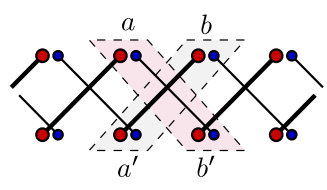

FIG. 2. (a) Representation of a QCA as a bilayer product of nearest neighbor unitaries, with possibly different Hilbert-space bipartition for their inputs and outputs. Here the thick and dotted legs refer to virtual local Hilbert spaces at even and odd sites in the hidden layer, respectively. (b) CJS $|U\rangle$ defined in Eq. (4) and the entanglement bipartition related to the index. (c) Relevant CJS used to derive the entropy expression (5) and the main result (1). (d) Demonstration of Eq. (5) for a general representative QCA with index $\log (p / q)$ consisting of the right and left translations of local Hilbert spaces $\mathbb{C}^{p}$ (red circles) and $\mathbb{C}^{q}$ (blue circles), respectively. where $\quad u_{2 x, 2 x+1}: \mathbb{C}^{d_{2 x}} \otimes \mathbb{C}^{d_{2 x+1}} \rightarrow \mathbb{C}^{d_{2 x}^{\prime}} \otimes \mathbb{C}^{d_{2 x+1}^{\prime}} \quad$ and $v_{2 x-1,2 x}: \mathbb{C}^{d_{2 x-1}^{\prime}} \otimes \mathbb{C}^{d_{2 x}^{\prime}} \rightarrow \mathbb{C}^{d_{2 x-1}} \otimes \mathbb{C}^{d_{2 x}}$ are unitaries, with input and output on two adjacent blocked sites, respectively. Note that the local Hilbert space dimension $d_{x}^{\prime}$ in the "hidden" layer is generally not equal to $d_{x}$, but they must satisfy $d_{x}^{\prime} d_{x+1}^{\prime}=d_{x} d_{x+1} \quad \forall x=1,2, \ldots, 2 l$, implying $d_{2 x}^{\prime} / d_{2 x}=d_{2 x-1} / d_{2 x-1}^{\prime}$ is a constant independent of $x=1,2, \ldots, l$. The index of $U$ is defined as the logarithm of this constant [22,23]:

$$
\text { ind } \equiv \log \frac{d_{2 x}}{d_{2 x}^{\prime}} \in \log \mathbb{Q}^{+},
$$

which was proven to be stable against different ways of blocking and under continuous deformations. In other words, this index is a topological invariant of $U$.

Equivalent formulation of the index.-As a starting point, we show that the index (3) can be expressed exactly as an entanglement entropy difference between two reduced states of the vectorized operator $|U\rangle$, technically known as the Choi-Jamiołkowski state (CJS) [40]:

$$
|U\rangle \equiv(U \otimes \mathbb{I})|I\rangle,
$$

where $|I\rangle \equiv d^{-N / 2}\left(\sum_{j=1}^{d}|j j\rangle\right)^{\otimes N} \quad$ is the maximally entangled state between two copies of the entire Hilbert space and $\mathbb{I} \equiv \mathbb{1}^{\otimes N}$ is the global identity acting on the auxiliary copy. As shown in Fig. 2(b), if we pick up two adjacent segments $a$ and $b$ with $\min \{|a|,|b|\} \geq r(|a|$ is the number of sites in $a$ ) [41], irrespective of their locations [42], the index turns out to be

$$
\text { ind }=\frac{1}{2}\left[S_{\alpha}\left(\rho_{a b^{\prime}}\right)-S_{\alpha}\left(\rho_{a^{\prime} b}\right)\right],
$$

where $\rho_{A} \equiv \operatorname{Tr}_{\bar{A}}[|U\rangle\langle U|]\left(A=a b^{\prime}, a^{\prime} b\right.$ and $\bar{A}$ is the complement of $A$ ) and $S_{\alpha}(\rho) \equiv(1-\alpha)^{-1} \log \operatorname{Tr}\left[\rho^{\alpha}\right]$ can be an arbitrary Rényi entropy. Hereafter, we denote by $a^{\prime}$ the ancillary qudits associated with $a$, and analogously for other regions. To show Eq. (5), we can take a specific bilayer representation such that $a$ and $b$ are blocked into an even and odd site, respectively. Then we consider the CJS shown in Fig. 2(c) corresponding to $\left(v_{L, a} \otimes v_{b, R}\right) u_{a, b}$, so that its reduced state on $L a a^{\prime} b b^{\prime} R$ coincides with that of $|U\rangle$. Importantly, here $L$ and $R$ are finite regions next to $a, b$ with $|L|,|R| \geq r$, cf. Fig. 2(c). Since this is a pure state, we have

$$
S_{\alpha}\left(\rho_{L^{\prime} a^{\prime} b R}\right)=S_{\alpha}\left(\rho_{L a b^{\prime} R^{\prime}}\right)
$$

under the bipartition $L^{\prime} a^{\prime} b R \sqcup L a b^{\prime} R^{\prime}$. By directly contracting the tensor, we obtain $\rho_{L^{\prime} a^{\prime} b R}=\mathbb{1}_{L^{\prime}} / d_{L}^{\prime} \otimes \rho_{a^{\prime} b R}$ and $\rho_{L a b^{\prime} R^{\prime}}=\rho_{L a b^{\prime}} \otimes \mathbb{1}_{R^{\prime}} / d_{R}^{\prime}$. Tracing out the auxiliary part of $|U\rangle$ except for $a^{\prime}$, we can consider $\rho_{a^{\prime} b R}$ as the reduced state of $\left(U \otimes \mathbb{1}_{a^{\prime}}\right)\left(\left|I_{a a^{\prime}}\right\rangle\left\langle I_{a a^{\prime}}\right| \otimes d_{a} \mathbb{I}_{\bar{a}} / d^{N}\right)\left(U^{\dagger} \otimes \mathbb{1}_{a^{\prime}}\right)\left(\left|I_{a a^{\prime}}\right\rangle\right.$ is the maximally entangled state between $a$ and $a^{\prime}$ ), which is 
supported on $L a a^{\prime} b$. This implies $\rho_{a^{\prime} b R}=\rho_{a^{\prime} b} \otimes \mathbb{1}_{R} / d_{R}$. Similarly, we can show $\rho_{L a b^{\prime}}=\mathbb{1}_{L} / d_{L} \otimes \rho_{a b^{\prime}}$. Therefore, $\rho_{L^{\prime} a^{\prime} b R}$ and $\rho_{L a b^{\prime} R^{\prime}}$ turn out to be supported on $a^{\prime} b$ and $a b^{\prime}$, respectively:

$$
\begin{aligned}
& \rho_{L^{\prime} a^{\prime} b R}=\frac{\mathbb{1}_{L^{\prime}}}{d_{L}^{\prime}} \otimes \rho_{a^{\prime} b} \otimes \frac{\mathbb{1}_{R}}{d_{R}}, \\
& \rho_{L a b^{\prime} R^{\prime}}=\frac{\mathbb{1}_{L}}{d_{L}} \otimes \rho_{a b^{\prime}} \otimes \frac{\mathbb{1}_{R^{\prime}}}{d_{R}^{\prime}} .
\end{aligned}
$$

Substituting Eq. (7) into Eq. (6) and recalling the definition of index in Eq. (3), we end up with Eq. (5).

It is instructive to test Eq. (5) for the QCA shown in Fig. 2(d), which are the simplest representatives with ind $=\log (p / q)$. This example provides a nice illustration of the index as a measure of the chirality of quantum information flow.

Before proceeding, we compare our result against different previous reformulations of ind in the literature. First, one can show [42] that Eq. (5) is equivalent to the "chiral mutual information" introduced in Ref. [31], which is defined in terms of some local ancillary degrees of freedom. We stress, however, that the derivation presented there is completely different from ours. In particular, we will see that our formalism naturally allows us to make a connection with different properties of the evolution operator. Second, the Rényi-2 version of Eq. (5) is easily seen to coincide with the original definition in Ref. [22] based on algebra overlaps [42].

Proof for the entanglement lower bound.-Before proving Eq. (1) for general Rényi- $\alpha$ entropies, we observe that one could show directly its validity for $\alpha=1$, i.e., the case of von Neumann entropy. Considering a segment with size larger than $2 r$ as the subsystem $A$, we can bipartite it into two adjacent segments $A=a \sqcup b$ with $\min \{|a|,|b|\} \geq r$, so that the entropy formula (5) is valid. Since the von Neumann entropy satisfies the triangle inequality [44], which follows from subadditivity, we have

$$
S\left(\rho_{a b a^{\prime} b^{\prime}}\right) \geq\left|S\left(\rho_{a b^{\prime}}\right)-S\left(\rho_{a^{\prime} b}\right)\right|=2 \mid \text { ind } \mid .
$$

Using monotonicity in $\alpha$ of $S_{\alpha}$, we immediately get that the bound is satisfied for $\alpha \leq 1$. Unfortunately, this proof cannot be extended to $\alpha>1$, since the Rényi entropies do not satisfy subadditivity in general [45].

To prove the general case, we should make further use of some nice properties of $\rho_{A A^{\prime}}$ as a reduced state of a pure CJS. To this end, let us return to the state shown in Fig. 2(c) and take a different bipartition $a b a^{\prime} b^{\prime} \sqcup L R L^{\prime} R^{\prime}$, obtaining

$$
S_{\alpha}\left(\rho_{a b a^{\prime} b^{\prime}}\right)=S_{\alpha}\left(\rho_{L R L^{\prime} R^{\prime}}\right)=S_{\alpha}\left(\rho_{L L^{\prime}}\right)+S_{\alpha}\left(\rho_{R R^{\prime}}\right),
$$

where we have used $\rho_{L R L^{\prime} R^{\prime}}=\rho_{L L^{\prime}} \otimes \rho_{R R^{\prime}}$. This relation follows from the fact that $U$ is a QCA [21], and can be understood from the vanishing correlation for two arbitrary observables supported on these two regions [42]. While the Rényi entropies do not satisfy subadditivity, they still satisfy the weak subadditivity [46]:

$$
S_{\alpha}\left(\rho_{L L^{\prime}}\right) \geq \max \left\{S_{\alpha}\left(\rho_{L}\right)-S_{0}\left(\rho_{L^{\prime}}\right), S_{\alpha}\left(\rho_{L^{\prime}}\right)-S_{0}\left(\rho_{L}\right)\right\},
$$

where $S_{0}(\rho) \equiv \log (\operatorname{rank} \rho)$ is the max entropy. This inequality follows from the nondecreasing property of Rényi entropies upon arbitrary unital channels [46]. Note that $\rho_{L}$ and $\rho_{L^{\prime}}$ are both maximally mixed and thus their Rényi entropies coincide with the maximum possible values $\log d_{L}$ and $\log d_{L}^{\prime}$, respectively. Therefore, we obtain $S_{\alpha}\left(\rho_{L L^{\prime}}\right) \geq \mid$ ind $\mid$. Similarly, we have $S_{\alpha}\left(\rho_{R R^{\prime}}\right) \geq$ ind $\mid$ and thus $S_{\alpha}\left(\rho_{a b a^{\prime} b^{\prime}}\right) \geq 2 \mid$ ind $\mid$, which completes the proof of the main result.

Obviously, the bound is tight for all the Rényi entropies for $\mid$ ind $\mid \in \log \mathbb{Z}^{+}$since they are saturated by left or right translations. What is less clear is whether the bound is tight for general ind $\in \log \mathbb{Q}^{+}$. At least for the Rényi- $\infty$ entropy, which gives the strongest version of Eq. (1) for a given QCA, we can readily construct an example which saturates the bound. To this end, we take $u: \mathbb{C}^{p} \otimes \mathbb{C}^{q} \rightarrow \mathbb{C}^{q} \otimes \mathbb{C}^{p}$ (with $q>p$ ) in Fig. 1(c) to be

$$
u=\sum_{m, n=1}^{p}|m n\rangle\left\langle m n\left|+\sum_{m=1}^{p} \sum_{n=p+1}^{q}\right| n m\right\rangle\langle m n| .
$$

This construction is reminiscent of the AKLT state [47], in the sense that it is essentially an assembly of dis joint unitaries but becomes correlated upon recombinations of subsystems. See Fig. 1(d) for a demonstration for $(p, q)=(2,3)$. On the other hand, since $S_{\alpha}>S_{\infty}$ for any finite $\alpha$ and $S_{\infty} \in \log \left(\mathbb{Q} \backslash \mathbb{Z}^{+}\right)$, the bound is not tight for any noninteger index and $\alpha<\infty$. Identifying a tight bound for the most general case thus remains an open problem.

One immediate and important implication of our main result is that it rigorously rules out, for QCA with nonzero index, the possibility of MBL, which implies a logarithmic growth of the entanglement of the evolution operator [48]. More generally, for nonzero index, any diffusive behavior characterized by a sublinear growth of the Rényi entropies, as recently demonstrated for random circuits with a diffusive charge [49,50], is forbidden. This is because the index is additive upon compositions [22] so the operator entanglement entropy after $t$ time steps is lower bounded by [51]

$$
S_{\alpha}(t) \geq 2 \mid \text { ind } \mid t,
$$

implying a linear growth. When specified to the case $\alpha=2$, this result also allows us to make a precise connection to quantum chaos, due to the known relation between $S_{2}(t)$ and the average of infinite-temperature OTOCs [39]: 


$$
\overline{\left\langle U^{t} O_{A}\left(U^{\dagger}\right)^{t} O_{\bar{A}} U^{t} O_{A}\left(U^{\dagger}\right)^{t} O_{\bar{A}}\right\rangle_{\beta}=0}=e^{-S_{2}(t)} .
$$

Here the lhs denotes the normalized sum over the elements $O_{A}, O_{\bar{A}}$ of a complete basis of observables supported in $A$, $\bar{A}$, respectively [39]. When combined with Eq. (12), we immediately obtain that a nonzero index implies exponential decay of the averaged OTOC. Once again, this is not consistent with a MBL evolution, because in this case it has been shown that OTOCs exhibit a power-law decay $[52,53]$. Finally, we remark that there is no universal lower bound for the (state) entanglement growth of an initial product state evolved by a QCA, as it can be seen in the simple case of translations.

Experimental relevance.-One important motivation to consider Rényi entropies is their accessibility in state-ofthe-art quantum simulation experiments [54-57]. Thanks to the entropy reformulation (5) of the index, all the quantities in our main result (1) are in principle measurable.

Let us explain the protocol to measure Rényi- $n$ entropies with $n \in \mathbb{N}$. We can straightforwardly generalize the strategies in Refs. [58-60] for quantum states to operators by vectorizing the latter into their CJSs. To measure $S_{n}$ of a bipartite state $\rho_{A B}=\left|\Psi_{A B}\right\rangle\left\langle\Psi_{A B}\right|$, the essential idea is to make use of $\operatorname{Tr}\left[\rho_{A}^{n}\right]=\operatorname{Tr}\left[\mathbb{T}_{A} \rho_{A B}^{\otimes n}\right]$, where $\mathbb{T}_{A}$ denotes the translation operator of subsystems $A$, which can be implemented by a sequence of SWAP unitaries [cf. Fig. 3(a)]. The value of $S_{n}$ can then be extracted by performing an interferometric measurement on an ancilla qubit after a sequence of controlled-SWAP gates as shown in Fig. 3(b) [58]. For our specific purpose of measuring the operator entanglement and the index, we should choose $A$ to be $a a^{\prime} b b^{\prime}, a b^{\prime}$, and $a^{\prime} b$, where $a$ and $b$ are adjacent segments whose sizes can be as small as the range of the QCA. In a proof-of-principle experiment, it would be good enough to construct a translation of qubits with $N=4$ and $r=1$, which may be implemented by 3 SWAP gates, so that we can set $|a|=1$ and $|b|=1$, and measure the Rényi-2 entropy (a)

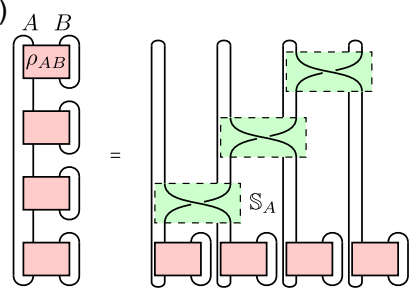

(b) $|0\rangle$

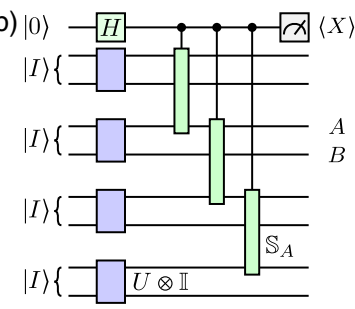

FIG. 3. (a) Graphic representation of $\operatorname{Tr}\left[\rho_{A}^{n}\right]=\operatorname{Tr}\left[\mathbb{T}_{A} \rho_{A B}^{\otimes n}\right]$ with $\mathbb{T}_{A}=\mathbb{S}_{A}^{[n-1, n]} \ldots \mathbb{S}_{A}^{[2,3]} \mathbb{S}_{A}^{[1,2]}$, where $\mathbb{S}_{A}^{[j, k]}$ is a sWAP over the $j$ th and $k$ th copies of subsystem $A$. (b) Experimental setup for measuring the operator entanglement Rényi- $n$ entropy as well as the index. Here $\mathbb{S}_{A}$ 's are controlled by the ancilla qubit, $H=(X+Z) / \sqrt{2}$ is the Hadamard gate, $|I\rangle$ 's are global maximally entangled states which become $|U\rangle$ upon the action of $U$, and $A$ is a subsystem of interest, whose choice depends on which quantity (index or operator entanglement) we would like to measure. so that we only need a single controlled-SWAP gate acting on 2 (index) or 4 (operator entanglement) pairs of qubits. This minimal setup should be accessible by many current experimental platforms, such as trapped ions [61], superconducting qubits [62], and Rydberg-atom arrays [63]. Note that there is also a more sophisticated method of measuring Rényi- $n$ entropies based on random quenches [64,65] which has also been experimentally realized [66] and should also be applicable to measuring operator entanglement and the index. As a final remark, we mention that one can further reduce the experimental cost in state preparation by taking advantage of the idea in Ref. [31]. That is, we may only introduce a few ancillas covering the subsystem, while obtaining the same measurement results.

Stability against exponential tails.-Trivial (nontrivial) QCA have been used to approximate the (edge) dynamics of 1D Floquet unitaries [67] (2D chiral Floquet MBL phases [28]) governed by time-periodic local Hamiltonians satisfying the Lieb-Robinson bound [68-70]. The QCA approximation should thus be precise up to some exponential tails outside the light cone. This motivates us to analyze how our main result (1) is modified by such small deviation from QCA.

To avoid the problem of defining the index for quasilocal unitaries with exponential tails, which remains an open problem [71], we restrict ourselves to consider specific quasilocal unitaries that are range- $r$ QCA followed by finite-time evolutions of local Hamiltoniains, i.e.,

$$
U=\hat{T} e^{-i \int_{0}^{T} d t H(t)} U_{\mathrm{QCA}},
$$

where $\hat{T}$ denotes the time ordering, $H(t)=\sum_{j=1}^{N} h_{j}(t)$ with $h_{j}(t)$ supported locally near $j$ and $h \equiv \max _{j, t}\left\|h_{j}(t)\right\|$ is finite. This setup has been used in several previous studies [28,31]. Setting the rhs of Eq. (1) as the index of $U_{\mathrm{QCA}}$, we would like to know whether the inequality can be violated and, if yes, to what extent.

We can give an explicit example where the inequality is violated by choosing $U_{\mathrm{QCA}}$ to be the right translation $\mathbb{T}$ and $H(t)=h \mathbb{S}^{\left[j_{t}, j_{t}+1\right]}$, where $j_{t}=\lfloor t|A| / T\rfloor$ and $A=$ $[0,|A|-1]$ is the subsystem of interest, while $\mathbb{S}^{[j, k]}$ is the SWAP operator between sites $j$ and $k$. This exactly solvable construction is inspired by the fact that $A A^{\prime}$ and $\bar{A} \bar{A}^{\prime}$ can be exactly disentangled for $T=\pi|A| /(2 h)$, so one may expect the Hamiltonian evolution is still a disentangler for a finite $T \ll|A|$. Indeed, we find that Eq. (1) is violated for all $\alpha>0$, with the largest violation being 2 ind $-S_{\infty}=\log \left[1+\left(d^{2}-1\right) \epsilon\right]>0$, where $\epsilon=$ $\sin ^{2|A|}(h T /|A|)$ scales as $e^{-\mathcal{O}(|A| \log |A|)}$ for large $|A|$, which also determines the scaling behavior of the violation.

In fact, the above example serves as a qualitatively worst case. That is, we can prove that for any local-Hamiltonian evolution, the order of the violation of Eq. (1) can never be larger than $e^{-\mathcal{O}(|A| \log |A|)}$ [42], and thus vanishes superexponentially in the thermodynamic limit. The proof 
involves a technique in Ref. [67] for approximating Hamiltonian dynamics by quantum circuits and a careful optimization of the Lieb-Robinson bound [72,73]. This rigorous derivation implies the stability of our main result and significantly widens its range of applicability. For example, we can rule out any sublinear entanglementgrowth behavior for the evolution operator $e^{-i H T} \mathbb{T}$ (thus forbidding MBL features), even if the local Hamiltonian $H$ is in the deep MBL phase [28].

Summary and outlook.-We have derived a convenient local expression for the index of 1D QCA, and proved that any Rényi- $\alpha$ entropy of the evolution operator is lower bounded by twice the index. This rigorous bound rules out any sublinear entanglement-growth behavior in nontrivial QCA and might be interpreted as a lower bound on quantum chaos, as opposed to the Maldacena-ShenkerStanford upper bound [74]. Since the Rényi entropy is accessible in cutting-edge atomic, molecular and optical experiments, our results should be experimentally observable. We have also discussed the validity of our bound against deviations from QCA by exponential tails.

One immediate question for future work is how to tighten the bound for a general rational index and Rényi- $\alpha$ entropy with $\alpha<\infty$. Another natural direction to explore is the generalization to the symmetry-protected case [25]. Here we expect that a nonzero symmetryprotected index will give rise to a linear growth of the entropy, even for ind $=0$. Finally, it may also be interesting to consider generalizations to quantum channels with suitable locality properties [21].

We thank Andrew C. Potter for very helpful comments on the manuscript. Z. G. is supported by the Max-PlanckHarvard Research Center for Quantum Optics (MPHQ). L. P. acknowledges support from the Alexander von Humboldt foundation. J. I. C. acknowledges support by the EU Horizon 2020 program through the ERC Advanced Grant QENOCOBA No. 742102 and from the DFG (German Research Foundation) under Germany's Excellence Strategy-EXC-2111-390814868.

Note added.-Recently, a related work by Ranard et al. appeared [75], which also reported the entropy reformulation of the index. However, their main focus was to derive an index theory for quasilocal unitaries rather than exploring its connections with other dynamical properties. On the technical level, Ref. [75] only uses the von Neumann entropy for infinite (or finite open) chains.

[1] B. Schumacher and R. F. Werner, arXiv:quant-ph/0405174.

[2] T. Farrelly, Quantum 4, 368 (2020).

[3] P. Arrighi, Nat. Comput. 18, 885 (2019).

[4] A. Nahum, J. Ruhman, S. Vijay, and J. Haah, Phys. Rev. X 7, 031016 (2017).
[5] A. Nahum, S. Vijay, and J. Haah, Phys. Rev. X 8, 021014 (2018).

[6] C. W. von Keyserlingk, T. Rakovszky, F. Pollmann, and S. L. Sondhi, Phys. Rev. X 8, 021013 (2018).

[7] T. Rakovszky, F. Pollmann, and C. W. von Keyserlingk, Phys. Rev. X 8, 031058 (2018).

[8] V. Khemani, A. Vishwanath, and D. A. Huse, Phys. Rev. X 8, 031057 (2018).

[9] C. Sünderhauf, D. Pérez-García, D. A. Huse, N. Schuch, and J. I. Cirac, Phys. Rev. B 98, 134204 (2018).

[10] A. Chan, A. De Luca, and J. T. Chalker, Phys. Rev. Lett. 121, 060601 (2018).

[11] A. Chan, A. De Luca, and J. T. Chalker, Phys. Rev. X 8, 041019 (2018).

[12] A. J. Friedman, A. Chan, A. De Luca, and J. T. Chalker, Phys. Rev. Lett. 123, 210603 (2019).

[13] B. Bertini, P. Kos, and T. Prosen, Phys. Rev. Lett. 123, 210601 (2019).

[14] B. Bertini and L. Piroli, Phys. Rev. B 102, 064305 (2020).

[15] S. Xu and B. Swingle, Nat. Phys. 16, 199 (2020).

[16] P. W. Claeys and A. Lamacraft, Phys. Rev. Research 2, 033032 (2020).

[17] P. Arrighi, V. Nesme, and R. Werner, J. Comput. Syst. Sci. 77, 372 (2011).

[18] J. Haah, L. Fidkowski, and M. B. Hastings, arXiv:1812.01625.

[19] J. Haah, arXiv:1907.02075.

[20] M. Freedman and M. B. Hastings, Commun. Math. Phys. 376, 1171 (2020).

[21] L. Piroli and J. I. Cirac, Phys. Rev. Lett. 125, 190402 (2020).

[22] D. Gross, V. Nesme, H. Vogts, and R. F. Werner, Commun. Math. Phys. 310, 419 (2012).

[23] J. I. Cirac, D. Perez-Garcia, N. Schuch, and F. Verstraete, J. Stat. Mech. (2017) 083105.

[24] M. B. Şahinoğlu, S. K. Shukla, F. Bi, and X. Chen, Phys. Rev. B 98, 245122 (2018).

[25] Z. Gong, C. Sünderhauf, N. Schuch, and J. I. Cirac, Phys. Rev. Lett. 124, 100402 (2020).

[26] L. Piroli, A. Turzillo, S. K. Shukla, and J. I. Cirac, J. Stat. Mech. (2021) 013107.

[27] D. V. Else and C. Nayak, Phys. Rev. B 93, 201103(R) (2016).

[28] H. C. Po, L. Fidkowski, T. Morimoto, A. C. Potter, and A. Vishwanath, Phys. Rev. X 6, 041070 (2016).

[29] A. C. Potter and T. Morimoto, Phys. Rev. B 95, 155126 (2017).

[30] F. Harper and R. Roy, Phys. Rev. Lett. 118, 115301 (2017).

[31] B. R. Duschatko, P. T. Dumitrescu, and A. C. Potter, Phys. Rev. B 98, 054309 (2018).

[32] L. Fidkowski, H. C. Po, A. C. Potter, and A. Vishwanath, Phys. Rev. B 99, 085115 (2019).

[33] C. Zhang and M. Levin, Phys. Rev. B 103, 064302 (2021).

[34] Y. Liu, H. Shapourian, P. Glorioso, and S. Ryu, arXiv:2012.08384.

[35] S. H. Shenker and D. Stanford, J. High Energy Phys. 12 (2014) 046.

[36] S. H. Shenker and D. Stanford, J. High Energy Phys. 03 (2014) 067.

[37] D. A. Roberts, D. Stanford, and L. Susskind, J. High Energy Phys. 03 (2015) 051. 
[38] P. Zanardi, Phys. Rev. A 63, 040304(R) (2001).

[39] P. Hosur, X.-L. Qi, D. A. Roberts, and B. Yoshida, J. High Energy Phys. 02 (2016) 004.

[40] M.-D. Choi, Linear Algebra Appl. 10, 285 (1975).

[41] To be rigorous, we should also require $|a|+|b| \leq N-2 r$, although typically $|a|,|b| \sim \mathcal{O}(r) \ll N$ is relevant to both practical numerical calculations and experimental measurements. The same constraint appears for the validity of Eq. (1) and explains why the minimal experimental setup is $N=4$.

[42] See Supplemental Material at http://link.aps.org/ supplemental/10.1103/PhysRevLett.126.160601, which includes Ref. [43], for details.

[43] P. Hayden, R. Jozsa, D. Petz, and A. Winter, Commun. Math. Phys. 246, 359 (2004).

[44] M. A. Nielsen and I. L. Chuang, Quantum Computation and Information (Cambridge University Press, Cambridge, England, 2010).

[45] N. Linden, M. Mosonyi, and A. Winter, Proc. R. Soc. A 469, 20120737 (2013).

[46] W. van Dam and P. Hayden, arXiv:quant-ph/0204093.

[47] I. Affleck, T. Kennedy, E. H. Lieb, and H. Tasaki, Phys. Rev. Lett. 59, 799 (1987).

[48] T. Zhou and D. J. Luitz, Phys. Rev. B 95, 094206 (2017).

[49] Y. Huang, IOP SciNotes 1, 035205 (2020).

[50] T. Rakovszky, F. Pollmann, and C. W. von Keyserlingk, Phys. Rev. Lett. 122, 250602 (2019).

[51] To avoid undesired finite-size saturation, we may choose the subsystem size to be no smaller than $2 r t$ for a range- $r$ QCA at time $t$; alternatively, given subsystem size $|A|$, we should focus on a time interval up to $|A| /(2 r)$.

[52] R. Fan, P. Zhang, H. Shen, and H. Zhai, Sci. Bull. 62, 707 (2017).

[53] Y. Huang, Y.-L. Zhang, and X. Chen, Ann. Phys. (Amsterdam) 529, 1600318 (2017).

[54] R. Islam, R. Ma, P. M. Preiss, M. E. Tai, A. Lukin, M. Rispoli, and M. Greiner, Nature (London) 528, 77 (2015).

[55] A. M. Kaufman, M. E. Tai, A. Lukin, M. Rispoli, R. Schittko, P. M. Preiss, and M. Greiner, Science 353, 794 (2016).
[56] N. M. Linke, S. Johri, C. Figgatt, K. A. Landsman, A. Y. Matsuura, and C. Monroe, Phys. Rev. A 98, 052334 (2018).

[57] A. Lukin, M. Rispoli, R. Schittko, M. E. Tai, A. M. Kaufman, S. Choi, V. Khemani, J. Léonard, and M. Greiner, Science 364, 256 (2019).

[58] P. Horodecki and A. Ekert, Phys. Rev. Lett. 89, 127902 (2002).

[59] D. A. Abanin and E. Demler, Phys. Rev. Lett. 109, 020504 (2012).

[60] A. J. Daley, H. Pichler, J. Schachenmayer, and P. Zoller, Phys. Rev. Lett. 109, 020505 (2012).

[61] R. Blatt and C. F. Roos, Nat. Phys. 8, 277 (2012).

[62] A. A. Houck, H. E. Türeci, and J. Koch, Nat. Phys. 8, 292 (2012).

[63] H. Levine, A. Keesling, A. Omran, H. Bernien, S. Schwartz, A. S. Zibrov, M. Endres, M. Greiner, V. Vuletić, and M. D. Lukin, Phys. Rev. Lett. 121, 123603 (2018).

[64] A. Elben, B. Vermersch, M. Dalmonte, J. I. Cirac, and P. Zoller, Phys. Rev. Lett. 120, 050406 (2018).

[65] B. Vermersch, A. Elben, M. Dalmonte, J. I. Cirac, and P. Zoller, Phys. Rev. A 97, 023604 (2018).

[66] T. Brydges, A. Elben, P. Jurcevic, B. Vermersch, C. Maier, B. P. Lanyon, P. Zoller, R. Blatt, and C. F. Roos, Science 364, 260 (2019).

[67] T. J. Osborne, Phys. Rev. Lett. 97, 157202 (2006).

[68] E. H. Lieb and D. W. Robinson, Commun. Math. Phys. 28, 251 (1972).

[69] S. Bravyi, M. B. Hastings, and F. Verstraete, Phys. Rev. Lett. 97, 050401 (2006).

[70] B. Nachtergaele and R. Sims, Commun. Math. Phys. 265, 119 (2006).

[71] Very recently, this was partially solved in Ref. [75] for infinite and finite open chains, but remains unsolved for finite periodic systems discussed here.

[72] M. B. Hastings, arXiv:1008.5137.

[73] Z. Gong, N. Yoshioka, N. Shibata, and R. Hamazaki, Phys. Rev. A 101, 052122 (2020).

[74] J. Maldacena, S. H. Shenker, and D. Stanford, J. High Energy Phys. 08 (2016) 106.

[75] D. Ranard, M. Walter, and F. Witteveen, arXiv:2012.00741. 